\title{
Re-Exploration of the Sustainability of Traditional Village Spatial Development in The Epidemic Era - From the Perspective of Jiangnan Region, China
}

\author{
Xinqu Liu ${ }^{1, *}$, Yongfa $\mathrm{Wu}^{1}$, Junyang $\mathrm{Liu}^{2}$, and Zaiyi Liao ${ }^{3}$ \\ ${ }^{1}$ Gold Mantis School of architecture, Soochow University, Jiangsu 215123, China \\ ${ }^{2}$ School of Architecture and Planning, University of Auckland, 1010, New Zealand \\ ${ }^{3}$ The Faculty of Engineering and Architectural Science (FEAS), Ryerson University, ON M5B 2K3
}

\begin{abstract}
The global spread of the COVID-19 has given severely challenge to the contemporary human settlement environment. Traditional villages, as an essential human settlement, which has been significant in contemporary life. This article takes the traditional village space in the Jiangnan region, China as an example to analyse the positive interaction between humans and nature, health, and space in its traditional design. This research points out that in the traditional village space, healthy human settlements have two levels: "material form" and "internal sprite". In addition to focusing on the transformation, innovation, and application of the technology, designers should exceed the material level to seek non-material dialogue and integration, to explore the important role of inner spiritual art for healthy living. Finally, this research combine with the urban epidemic prevention function and pointed out that the symbiosis mechanism between urban and rural areas should be actively explored. The penetration and integration of urban and rural areas can better cure, and promote the sustainable protection, activation, utilization and innovation of traditional villages in the future.
\end{abstract}

\section{Introduction}

At the beginning of 2020 , the global outbreak of the COVID-19 broke the original stable social situation and the traditional thinking mode as a designer. This is a warning and an important opportunity for change. A healthy and harmonious human settlement has always been an important goal of human development, and it is also the unshakable foundation of life[1]. Traditional villages, as a typical living environment space for rural revitalization in China[2], are also the foundation and important practice model for contemporary healthy living activities. Although traditional villages lack advanced science and technology compared with urban spaces, the living wisdom that is harmonious with nature formed during long-term human settlement activities cannot be ignored. Therefore, analysing the advantages and disadvantages of the traditional village space under the emergent epidemic and its role in large-scale public health emergencies is of great significance to sustainable development. On the one hand, it is conducive to reflect on and improve the lack of contemporary human settlement space in public health security. Moreover, it can deeply reflect on the possibility of the transformation of traditional urban and rural pluralist values, so then to explore the contemporary urban-rural symbiosis mechanism to further promote contemporary rural revitalization. Therefore, this paper uses architectural typology[3,4], taking traditional villages in Jiangnan region as a typical case, analyses the important types and spiritual performances of healthy human settlements in their spaces. Finally, this research tries to explore the sustainable development of contemporary traditional villages through comparative research with contemporary urban space epidemic prevention.

\section{Spatial types and characteristics of Jiangnan traditional villages}

\subsection{Ecosystem}

\subsubsection{Taking water as the origin}

In terms of the developed water system and flat terrain in the Jiangnan region villages tend to grow towards the water, forming a village space dominated by water systems and river networks[5]. The village conforms to the distribution of water networks and roads and uses abundant water resources to form a powerful irrigation and transportation system. As the basic condition of traditional farming life, water act an important role in the traditional culture of Jiangnan. It is not only in ancient books such as "The Classic of Mountains and Seas"(called"山海经" in Chinese) [6] and " The Shi King"(called" 诗经" in

* Corresponding author: liuqicrystal@foxmail.com 
Chinese ) [7] that many traditional mythological images and gods are derived from water. Even in the square, temples, pagodas, and other sacrifice places also worship the water like the god. The diverse forms and flowing rhythm of the water system enrich the space of traditional villages and form corresponding design techniques, using water to form a dynamic and static artistic expression of water landscaping (see Figure1).

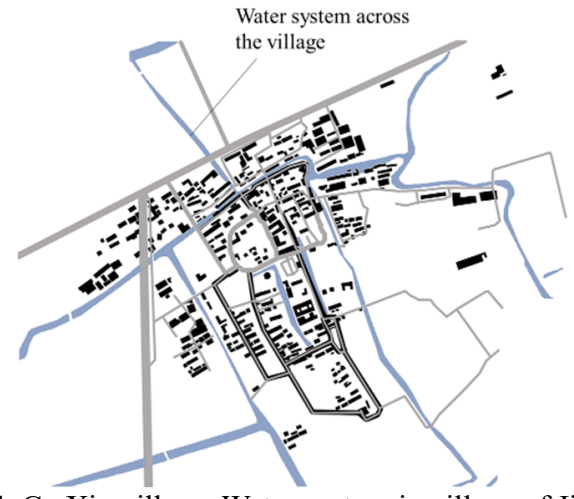

Fig1. Ge Xin village: Water system in village of Jiangnan region

\subsubsection{Take the mountain as a protective barrier}

This kind of traditional villages are mainly distributed in areas dominated by mountainous landforms in southern Anhui and northern Zhejiang $\left[^{8,9}\right]$. These villages are built on the basis of the mountain terrain. Therefore, the villages are mainly distributed at the foot of the mountain, between the mountains or the ridge, and are closely integrated with the terrain (see Figure2).

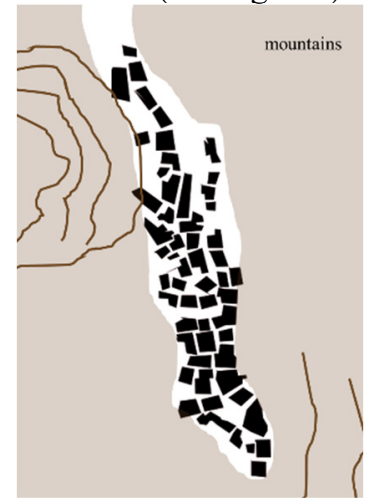

Fig2. Mu Ligong village: Mountain system around village of Jiangnan region

\subsubsection{The combination of mountains and waters}

The villages are mainly distributed in areas with both mountains and water. They are favourable geomorphic conditions for the formation of traditional villages in the Jiangnan region, surrounded by mountains and waters. The soil in the area is fertile, suitable for the growth of rice and other crops. In this drastic change, traditional craftsmen rely on Feng Shui principles to form a visual landscape axis, which shows the beauty of ecology by combining multiple natural elements $\left[{ }^{10}\right]$. The overall shape of the village is free and provides favourable conditions that are used to form a boundary traffic and water network system. (see Figure3).

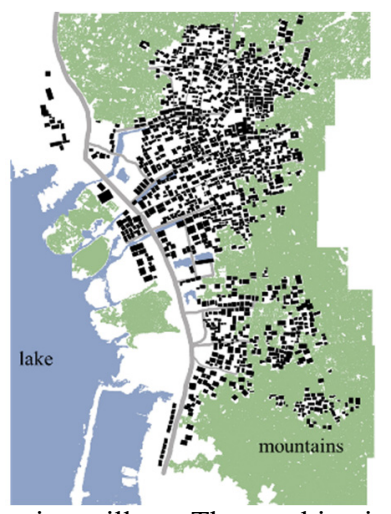

Fig3. Luxiang ancient village: The combination of mountains and waters in village of Jiangnan region

\subsection{Spatial pattern}

\subsubsection{Centripetal aggregation style}

This type of spatial pattern has formed one or more important spatial focal points in the natural development of the village, mainly composed of ancestral halls, temples, and partly composed of village squares or important landscape nodes (see Figure4). This kind of village spatial layout is tidier and more orderly, and the village core is prominent, which has a strong guiding effect on villager's behaviours. Inside the village, the village buildings and roads are spread out around the centre layer by layer, and the overall form is in a tightly gathered form.

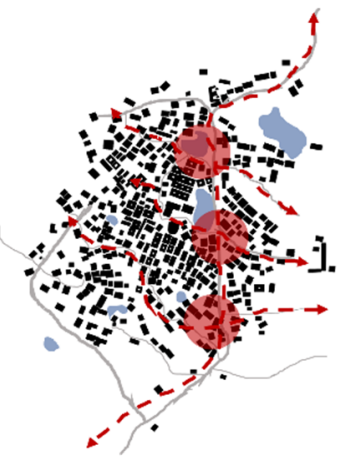

Fig4. Shang Wufang village: The centripetal aggregation style in village of Jiangnan region

\subsubsection{Reticular radiation style}

This type begins to radiate around a point in the village or an important public building or landscape node in the village as the centre. The shape presents the characteristics of spreading and radiating shapes, just like a spread net attached to the natural landscape (see Figure5). Traditionally, this type of village is affected by the degree of population concentration and is generally large in scale. Moreover, the form of the spatial pattern presents a flexible and outward-looking visual aesthetics, which as a whole highlight a yearning tension and inner vitality. 


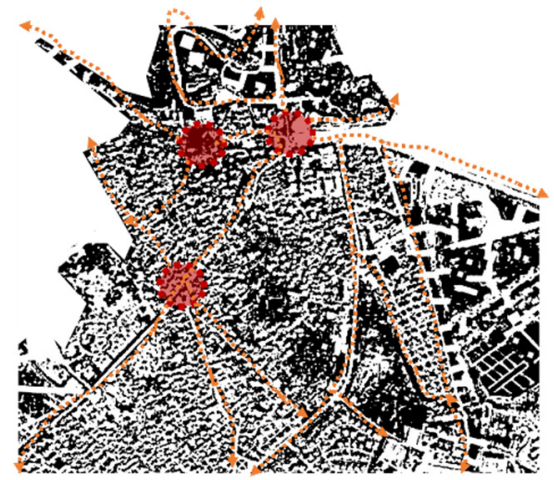

Fig5. Weng Xiang village: The reticular radiation style of Jiangnan region

\subsubsection{Free scatter style}

This type of village is generally located in an area with extreme topographical conditions or severe climatic conditions, which prevents the villages from being able to gather in a certain area on a large scale or restricts traditional construction techniques. Affected by this typical external condition, the village can only use roads as the main connection, and gather in a small area under suitable terrain conditions, showing a relatively scattered form (see Figure6).

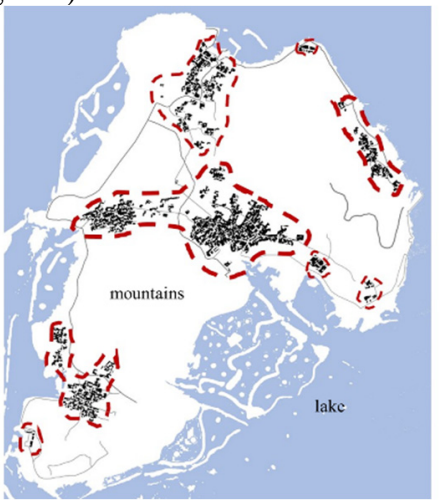

Fig6. San Shan village: The free scatter style of Jiangnan region

\subsection{Typical traditional residence}

\subsubsection{Waterfront residences}

Residences are built near the water, borrowing space to take space, usually by introducing water system or adjusting the microclimate. The scale of the housing is proportional to the size of the family and the economic situation. The architecture structure is mainly a combination of lifting beam and wearing bucket (see Figure7).
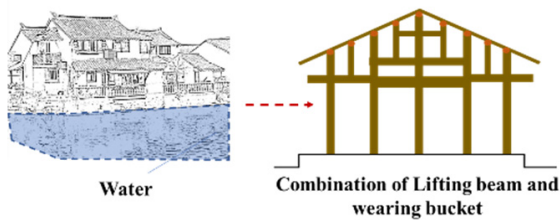

Fig7. The form and structure of waterfront residence

\subsubsection{Street-facing residence}

The residence takes the street orientation as the main space layout, free form, and realizes the purpose of saving land and optimizing space by using the terrain. The architecture structure is combination of lifting beam and wearing bucket or wearing bucket (see Figure8).

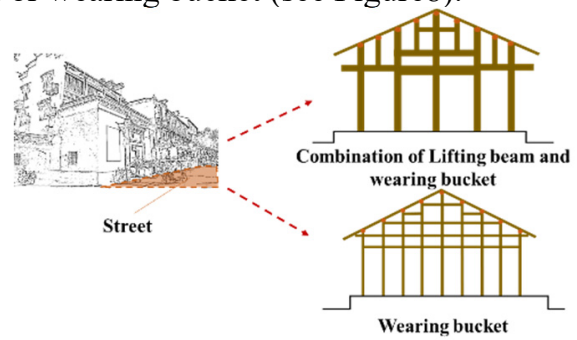

Fig8. The form and structure of street-facing residence

\subsubsection{Courtyard residence}

This type of residence is mostly built in a flat area, with courtyards that have unique themes as the main form of closed buildings. The structure of the building is mainly combination of lifting beam and wearing bucket or wearing bucket (see Figure9).

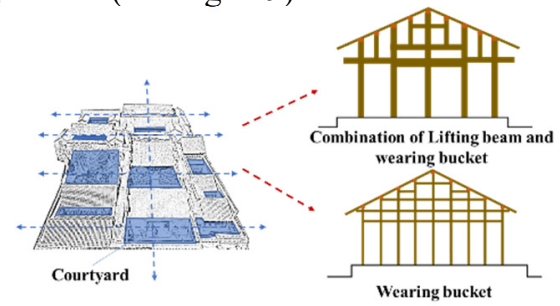

Fig9. The form and structure of Courtyard residence

\subsubsection{Chinese and Western combined dwellings / New Shikumen}

The residences are mainly distributed in economic development areas influenced by Western culture. The architectural form features prominent western elements, simplified facade, obvious functional distinction, clear traffic line. The building structure is brick-wood structure (see Figure10).

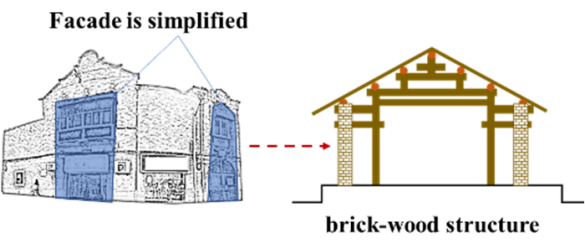

Fig10. The form and structure of Chinese and Western combined dwellings / New Shikumen

\subsubsection{Garden residence}

The dwellings were mainly built in the Ming and Qing dynasties, and were influenced by the historical scholars, and were built in the economically developed areas of Jiangnan. The structure of the building is mainly combination of lifting beam and wearing bucket (see Figure11). 


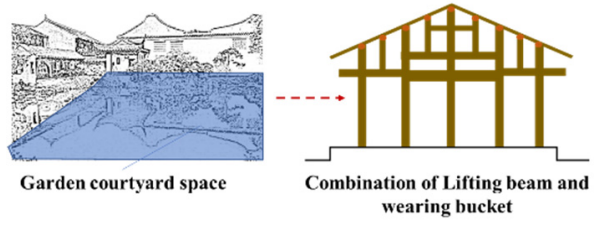

Fig11. The form and structure of Garden residence

\section{Jiangnan Traditional Village Space and Contemporary Healthy Human Habitat}

\subsection{Advantages of space epidemic prevention in traditional villages}

Jiangnan has had unique geographical advantages since ancient times. The humid climate and favourable landscape pattern have made the traditional villages ingeniously integrated with nature $\left.{ }^{11}\right]$. Compared with modern cities, traditional villages have low population density, are close to nature, and have open and flexible spaces. Therefore, the spatial structure of the countryside has less destructive power and influence on the natural environment, forming a circular and sustainable integration model of survival, production, and life. Under the influence of the epidemic environment, rural areas dominated by agriculture can ensure self-sufficiency in daily life without relying on external forces, and the cohesion of the rural population is more convenient for unified management under the impact of emergency security incidents.

\subsection{Traditional village space is weak in epidemic prevention}

In order to specifically study the thermal comfort of the natural environment of Jiangnan villages, this research used 186 questionnaires and 50 traditional residences as the survey samples to conduct a field survey on the thermal comfort of residences in the Jiangnan region. In terms of indoor comfort in four seasons (see Table1).

Table1. Thermal comfort analysis of Jiangnan traditional village residences

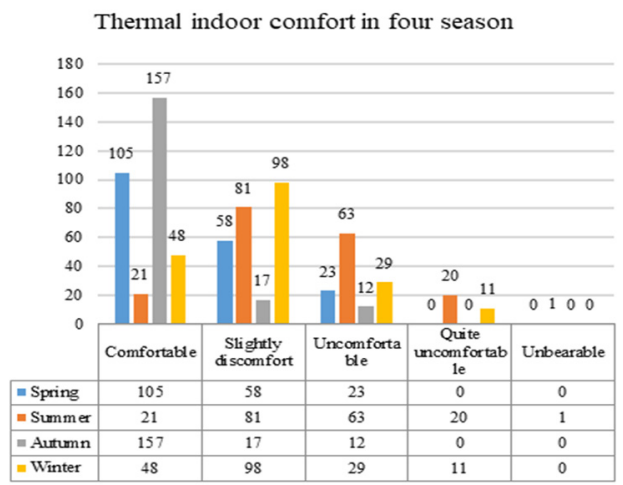

$56.4 \%$ of people feel comfortable indoors in spring, and $84.4 \%$ of people feel comfortable indoors in autumn. On the contrary, $43.5 \%$ of people feel slightly uncomfortable in summer, $33.8 \%$ feel uncomfortable, $10.8 \%$ feel very uncomfortable; in winter $52.6 \%$ feel slightly uncomfortable, $15.6 \%$ feel uncomfortable $5.9 \%$ of people feel very uncomfortable. Therefore, in Jiangnan traditional residences, most users think that the indoor comfort is comfortable in spring and autumn, but the indoor comfort is weak in summer and winter.

In order to accurately measure the thermal comfort in summer, the indoor and outdoor temperature, humidity and wind speed were measured in 50 residences without air-conditioning and other equipment in summer, and finally the comfort index of 50 households was calculated. The results show that the thermal comfort index of these residences in summer are all above 80 (see Table2). Comparing with the human body comfort index grading table, the range of comfort index felt by a normal human body is 50-70. Therefore, in summer, the comfort index of Jiangnan traditional residences far exceeds the normal comfort index of the human body. Consequently, the thermal comfort of traditional residences in Jiangnan still needs to be improved.

Table2. Analysis on the comfort of 50 residences in Jiangnan traditional village Analysis on the comfort of 50 residences

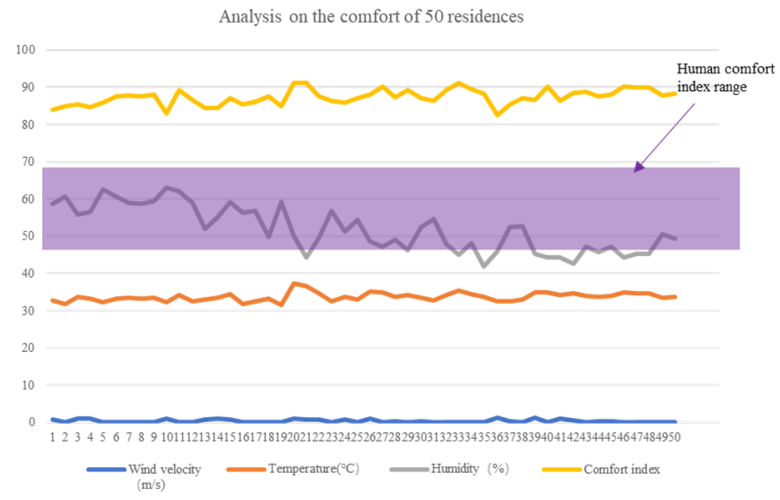

To sum up, although traditional villages provide a favourable ecological and healthy human settlement foundation and have strong natural restoration capabilities, they still expose many shortcomings. Due to the traffic congestion, the economic level of the village has been lagging behind that of the city for a long time, resulting in insufficient awareness of the residents of the public health environment, so they lack the ability to respond effectively to the impact of the epidemic.

\section{Urban-rural interaction under the epidemic}

\subsection{The symbiosis mechanism of urban and rural areas}

At the level of the energy field, there are multiple apparently interdependent energy elements between contemporary urban and rural areas, and the mutual influence and interaction between the two are realized through the flow or conversion of energy $\left.{ }^{12}\right]$. It mainly includes material field, information field, capital field, human field, technology field, etc. The ultimate goal is to achieve maximum resource sharing under limited material 
conditions. Taking Space field to effectively serve different types of contemporary communities the Jiangnan villages during the epidemic period as an example. Villages dominated by agriculture, farming, fisheries, etc. provide necessary living supplies for cities, while cities import new resources into the countryside. Science and technology and medical resources, while realizing the monitoring and resource exchange of urban and rural epidemics through platforms such as the internet and media.

At the spatial field level, urban and rural areas are important spaces for contemporary healthy human settlements. In the spatial framework, the transition from the natural environment to the physical environment of human settlements has been realized $\left[{ }^{13}\right]$. Different spatial elements reasonably constitute different spatial scales and have certain laws and logics. Therefore, the space field can serve different types of contemporary communities effectively. In this field, both urban and rural areas have their own spatial independence and form an organic whole. Because any changes in the environment and public space will affect the shock of the overall space system, it means that any reversal and disorder of spatial elements will cause the balance of urban and rural spaces to become unbalanced.

At the level of consciousness field, it is gradually formed under the combined action of urban and rural energy field and material field to guide urban and rural practitioners and users, and it also reacts to energy field and space field $\left[{ }^{14}\right]$. As an intangible product of thought, its power is deeper, and it is a comprehensive manifestation of the collective consciousness response. The transformation and expansion of the field of consciousness can directly and effectively feed back to the positive behaviours of practitioners and users, and it has a deeper guiding role in the contradiction of thinking in the two perspective of urban and rural areas. Finally, through the positive practice of behavioural activities, reasonable planning and improvement of the overall urban and rural system space are carried out, and the contradiction is gradually transformed into an effective driving force for development.

\subsection{Reconsideration on the contradictions and symbiosis of contemporary urban and rural areas}

For a long time, there have been conflicts between the poor and the rich, the slow and the fast, the new and the old that cannot be ignored between the urban and rural areas in Chinese society. Consequently, the social, consciousness and other multi-level conflicts have been produced, which restricts the possibility of mutual benefit and win-win in the development process of urban and rural areas, and has formed a limited understanding of their relationship for a long time. Through the analysis of the characteristics of urban and rural areas in the epidemic, it can be clearly seen that the shortcomings of cities are the advantages of the villages, while the disadvantages of the villages are the advantages of the city, which shows an obvious opposite complementary relationship (see
Figure12). If contemporary designers can consider the pros and cons of urban and rural space from a holistic perspective. Complementing each other's strengths between urban and rural areas can just make up for the lack of development, achieving healthy and sustainable development of urban and rural areas. The purpose is to improve the contradictions caused by the confrontation in the past, and realize the transformation from "urban-rural contradictions to urban-rural integration". By transforming the contradiction between urban and rural areas, it can help improve the resilience of urban and rural spaces. The symbiotic between urban and rural spaces provides more convenient and more diverse possibilities for future development.

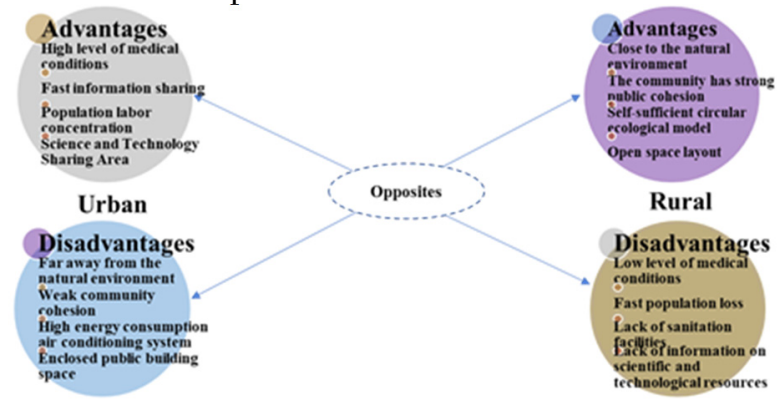

Fig12. Analysis of the opposite relationship between urban and rural areas under the epidemic

From the urban and rural system and antagonism to internalize into speculation, it is necessary to establish a "city-rural" integration concept. According to the characteristics and deficiencies of the urban-rural spatial relationship, a balance urban-rural relationship system can be constructed from four levels: co-construction, sharing, symbiosis, and co-creation (see Figure13).

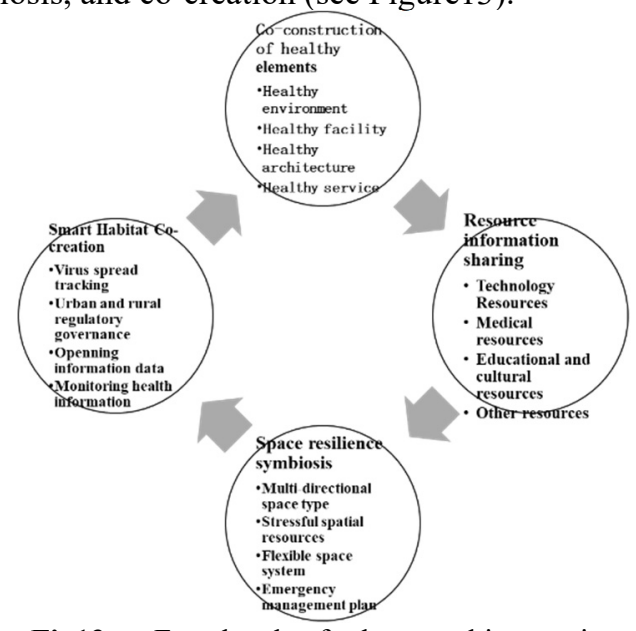

Fig13. Four levels of urban-rural integration

First, the joint construction of urban and rural health elements is to improve the weak links of the primary medical environment and facilities. Ensure the necessary hardware equipment in the entire disease prevention and control process, and build the medical space structure based on the principles of pathological transmission and the needs of healthy living. Gradually establish the foundation of a stable and healthy urban and rural area, and think deeply about the essential elements of a healthy and healthy circulation space according to the corresponding shortcomings, and realize the design requirements of healthy building space while paying attention to health psychology. Second, the sharing of 
urban and rural resource information, that is, the sharing of multiple resources such as technology, medical care, education and culture, maximizes the utilization of various resources. The use of various information platforms to serve different population structures enables the fair, open and free use of effective resource structures to achieve efficient production and construction while helping to balance social and economic imbalances. Third, the symbiosis of urban and rural resilience, which mainly cuts into the variability and flexibility of urban and rural spaces, and explores the possibility of transforming urban and rural static spaces into dynamics. It is based on the use of effective resources to actively construct a multi-functional and spatial multi-dimensional urban-rural integration model, which improves the stability of the society by ensuring the fluidity and variability of the human settlement environment under stress conditions. Fourth, co-creation of smart human settlements. That is, through the use of contemporary intelligent information technology to realize the monitoring of healthy human settlements and the development of a modern model of urban and rural integration. It is convenient for effective supervision and real-time monitoring of known diseases. And it also has a predictive effect on the emergence of unknown new viruses in the long-term development.

\section{Conclusion}

The large-scale spread of the COVID-19 has sounded a new round of alarm for us. Compared with this epidemic, contemporary chronic diseases such as obesity, diabetes, and depression have gradually eroded human health. The stressful and fast-paced living conditions, we have to reflect on whether rural space has played a positive role in human health in this process. According to the logic of historical development, with the advancement of science and technology, modern urban space should be able to solve many contradictions. However, it is a pity that the rapid urbanization construction has made the life of contemporary people a kind of "regression" $\left[{ }^{15}\right]$. Moreover, objective facts tell us that the "side effects" caused by the epidemic have obviously not faded away, and that problems will have a continuous impact on the human settlement environment for a period of time in the future. Therefore, as designers, we still need to improve vigilance and sensitivity.

Since China proposed rural revitalization in 2017 , the enthusiasm for protecting and developing the countryside has not faded, and the interactive relationship between the countryside itself and urban and rural areas is still in the exploratory stage. Through the in-depth analysis of the different feedback mechanisms of two typical human settlements under the epidemic situation, it can be clearly distinguished that the opposition between urban and rural areas has the possibility of complementary symbiosis. In addition, contemporary villages are facing a selfawakening, and the contradictory urban-rural values of multiple contradictions also need to be re-examined and effectively converted. However, urban-rural practices cannot be achieved just one day. Since the construction of the urban-rural relationship is the same as architectural design, there is a time gap between theory and practice, which leads to disconnection. In this epidemic, the display of forces from all parties has reduced this gap as much as possible, so there are many possibilities for future urbanrural integration. It is necessary to comprehensively consider its overall operating mode in space, and also to deeply consider the effectiveness of independent individuals.

\section{References}

1. Zeng Wei, Gao Xinyi, Zhao Yingjie. A Brief Analysis of "Healthy Human Settlements" Research Framework. J. Hum. Settlements West China 2, 3342 (2020).

2. Weng, L. Spatial Distribution of Traditional Chinese Villages and Factors Affecting Their Distribution. $J$. Landsc. Res. 11, 29-37 (2019).

3. Diane Ghirardo. The Architecture of the City. (The MIT Press, 1984).

4. Wang Lijun. Architectural typology. (Tianjin University Press, 2005).

5. Chinese, C., Town, W., Zhijian, G., Chang, G. \& Qiao, G. Z. Jiangnan: Views of a Contemporary Chinese Water Town. Cross-Currents East Asian Hist. Cult. Rev. 20, 127-135 (2016).

6. Birrell, A. The Classic of Mountains and Seas. (Penguin, 2000).

7. James Legge. The Shi King. (Oxford University Press, 1876).

8. Fenzhong, W. Eastern Residence Legend Zhejiang Dongyang Residence. (Tianjin: Tianjin University Press, 2008).

9. Bian, L. One thousand years of historical relics : the Huizhou Documents. J. Mod. Chinese Hist. 10, 248268 (2016).

10. Case, J. A., Liukeng, A., County, L., Huan, W. \& Zhongrong, M. I. N. Study on the Inheritance of Landscape Culture in Traditional Ancient Province. $J$. Landsc. Res. 7, 64-66 (2015).

11. Yi, C. Renovation of Traditional Water Villages in Jiangnan: a Casa study of Renovation Planning of Wenchang Village. J. Landsc. Res. 9, 15-17 (2017).

12. Matthias Naumann1;David Rudolphb. Conceptualizing rural energy transitions: Energizing rural studies, ruralizing energy research. J. Rural Stud. 73, 97-104 (2020).

13. Zhu, J.;Zhu M.;Xiao Y. Urbanization for rural development: Spatial paradigm shifts toward inclusive urban-rural integrated development in China. J. Rural Stud. 71, 94-103 (2019).

14. Cody, S. 'A new consciousness of the countryside'? Elite ruralism in contemporary China. Asian Anthropol. 18, 21-36 (2019).

15. Wang Dong. Countryside: As a Kind of Power of Critique and Thought. Archit. 6, 100-108 (2017). 